\title{
APPROXIMATE AMENABILITY OF SEGAL ALGEBRAS
}

\author{
MAHMOOD ALAGHMANDAN \\ (Received 10 July 2012; accepted 3 December 2012; first published online 18 July 2013) \\ Communicated by G. Willis
}

\begin{abstract}
In this paper we first show that for a locally compact amenable group $G$, every proper abstract Segal algebra of the Fourier algebra on $G$ is not approximately amenable; consequently, every proper Segal algebra on a locally compact abelian group is not approximately amenable. Then using the hypergroup generated by the dual of a compact group, it is shown that all proper Segal algebras of a class of compact groups including the $2 \times 2$ special unitary group, $\mathrm{SU}(2)$, are not approximately amenable.
\end{abstract}

2010 Mathematics subject classification: primary 46H20; secondary 43A20, 43A62, 46H10.

Keywords and phrases: approximately amenable Banach algebra, Segal algebra, abstract Segal algebra, locally compact abelian group, compact group, hypergroup, Leptin condition.

\section{Introduction}

The notion of approximate amenability of a Banach algebra was introduced by Ghahramani and Loy in [8]. A Banach algebra $\mathcal{A}$ is said to be approximately amenable if for every $\mathcal{A}$-bimodule $X$ and every bounded derivation $D: \mathcal{A} \rightarrow X$, there exists a net $\left(D_{\alpha}\right)$ of inner derivations such that

$$
\lim _{\alpha} D_{\alpha}(a)=D(a) \quad \text { for all } a \in \mathcal{A} \text {. }
$$

This is not the original definition but it is equivalent. In [8], it is observed that approximately amenable algebras have approximate identities; moreover, closed ideals with a bounded approximate identity and quotient algebras of approximate amenable Banach algebras are approximately amenable.

In this paper, we study the approximate amenability of proper abstract Segal algebras of Fourier algebras and, subsequently, Segal algebras on abelian groups and compact groups. The definition of Segal algebras will be given in Section 2.

The author was supported by a Dean's PhD scholarship at the University of Saskatchewan.

(c) 2013 Australian Mathematical Publishing Association Inc. 1446-7887/2013 \$16.00 
Approximate amenability of Segal algebras has been studied in several papers. Dales and Loy, in [4], studied approximate amenability of Segal algebras on $\mathbb{T}$ and $\mathbb{R}$. They showed that certain Segal algebras on $\mathbb{T}$ and $\mathbb{R}$ are not approximately amenable. It was further conjectured that no proper Segal algebra on $\mathbb{T}$ is approximately amenable. Choi and Ghahramani, in [3], have shown the stronger fact that no proper Segal algebra on $\mathbb{T}^{d}$ or $\mathbb{R}^{d}$ is approximately amenable. We extend the result of Choi and Ghahramani to apply to all locally compact abelian groups, not just $\mathbb{T}^{d}$ and $\mathbb{R}^{d}$. Our approach, like that of Choi-Ghahramani and Dales-Loy, is to apply the Fourier transform and work with abstract Segal subalgebras of the Fourier algebra of a locally compact abelian group.

In fact, we prove a more general result in Section 2: when $G$ is an amenable locally compact group, no proper Segal subalgebra of the Fourier algebra is approximately amenable. The proof makes use of the equivalence between amenability of $G$ and the so-called Leptin condition on $G$.

In the rest of the paper, we try to apply similar tools, this time for compact groups. In Section 3 our idea is to view the dual of compact groups as a discrete hypergroup. The Fourier space of hypergroups is studied as well. In Section 4, we introduce an analogue for hypergroups of the classical Leptin condition, and show that this holds for certain examples. In Section 5, we apply these tools to show that for a class of compact groups including SU(2), every proper Segal algebra is not approximately amenable.

\section{Abstract Segal algebras of Fourier algebra on amenable groups}

Let $\mathcal{A}$ be a commutative Banach algebra. We denote by $\sigma(\mathcal{A})$ the spectrum of $\mathcal{A}$ which is also called the maximal ideal space or character space of $\mathcal{A}$. A commutative Banach algebra $\mathcal{A}$ is called regular, if for every $\phi$ in $\sigma(\mathcal{A})$ and every open neighbourhood $U$ of $\phi$ in the Gelfand topology, there exists an element $a \in \mathcal{A}$ such that $\phi(a)=1$ and $\psi(a)=0$ for each $\psi \in \sigma(\mathcal{A}) \backslash U$. Using Gelfand representation theory, for a commutative semisimple regular Banach algebra $\mathcal{A}$, it can be viewed as an algebra of continuous functions on its spectrum, $\sigma(\mathcal{A})$. For each $a \in \mathcal{A}$, $\operatorname{supp}(\hat{a})$ is defined as the support of the function $\hat{a}$.

Let $G$ be a locally compact group, equipped with a fixed left Haar measure $\lambda$. The Fourier algebra of $G$ was defined and studied by Eymard in [6]. In the following lemma, we summarize the main features of the Fourier algebra, denoted by $A(G)$, which we need here. We denote by $C_{c}(G)$ the space of continuous, compactly supported, complex-valued functions on $G$.

Lemma 2.1. Let $G$ be a locally compact group and $K$ be a compact subset of $G$, and let $U$ be an open subset of $G$ such that $K \subset U$. For each relatively compact open set $V$ such that $K V V^{-1} \subseteq U$, we can find $u_{V} \in A(G) \cap C_{c}(G)$ such that:

(1) $u_{V}(G) \subseteq[0,1]$;

(2) $\left.u_{V}\right|_{K} \equiv 1$; 
(3) $\operatorname{supp}\left(u_{V}\right) \subseteq U$;

(4) $\left\|u_{V}\right\|_{A(G)} \leq(\lambda(K V) / \lambda(V))^{\frac{1}{2}}$.

The existence of $u_{V} \in A(G) \cap C_{c}(G)$ satisfying (1)-(3) is proved in [6, Lemma 3.2]. Also based on the proof of [6, Lemma 3.2] and the definition of the norm of $A(G)$, one can show (4).

Since $\sigma(A(G))=G$, Lemma 2.1 shows that $A(G)$ is a commutative semisimple regular algebra.

We say that the Banach algebra $\left(\mathcal{B},\|\cdot\|_{\mathcal{B}}\right)$ is an abstract Segal algebra of a Banach $\operatorname{algebra}\left(\mathcal{A},\|\cdot\|_{\mathcal{A}}\right)$ if:

(1) $\mathcal{B}$ is a dense left ideal in $\mathcal{A}$;

(2) there exists $M>0$ such that $\|b\|_{\mathcal{A}} \leq M\|b\|_{\mathcal{B}}$ for each $b \in \mathcal{B}$;

(3) there exists $C>0$ such that $\|a b\|_{\mathcal{B}} \leq C\|a\|_{\mathcal{A}}\|b\|_{\mathcal{B}}$ for all $a, b \in \mathcal{B}$.

If $\mathcal{B}$ is a proper subalgebra of $\mathcal{A}$, we call it a proper abstract Segal algebra of $\mathcal{A}$.

Let $G$ be a locally compact group. A linear subspace $S^{1}(G)$ of $L^{1}(G)$, the group algebra of $G$, is said to be a Segal algebra on $G$, if it satisfies the following conditions:

(1) $S^{1}(G)$ is dense in $L^{1}(G)$;

(2) $S^{1}(G)$ is a Banach space under some norm $\|\cdot\|_{S^{1}}$ and $\|f\|_{S^{1}} \geq\|f\|_{1}$ for all $f \in S^{1}(G)$;

(3) $S^{1}(G)$ is left translation invariant and the map $x \mapsto L_{x} f$ of $G$ into $S^{1}(G)$ is continuous, where $L_{x} f(y)=f\left(x^{-1} y\right)$;

(4) $\left\|L_{x} f\right\|_{S^{1}}=\|f\|_{S^{1}}$ for all $f \in S^{1}(G)$ and $x \in G$.

Note that every Segal algebra on $G$ is an abstract Segal algebra of $L^{1}(G)$ with convolution product. Similarly, we call a Segal algebra on $G$ proper if it is a proper subalgebra of $L^{1}(G)$. For the sake of completeness we will give some examples of Segal algebras.

- $\quad$ Let $\mathcal{L} A(G):=L^{1}(G) \cap A(G)$ and $\|h\|\|:=\| h\left\|_{1}+\right\| h \|_{A(G)}$ for $h \in \mathcal{L} A(G)$. Then $\mathcal{L} A(G)$ with norm $\||\cdot|\|$ is a Banach space; this space was studied extensively by Ghahramani and Lau in [7]. They have shown that $\mathcal{L} A(G)$ with the convolution product is a Banach algebra called the Lebesgue-Fourier algebra of $G$; moreover, it is a Segal algebra on the locally compact group $G . \mathcal{L} A(G)$ is a proper Segal algebra on $G$ if and only if $G$ is not discrete.

Also, $\mathcal{L} A(G)$ with pointwise multiplication is a Banach algebra and even an abstract Segal algebra of $A(G)$. Similarly, $\mathcal{L} A(G)$ is a proper subset of $A(G)$ if and only if $G$ is not compact.

- $\quad$ The convolution algebra $L^{1}(G) \cap L^{p}(G)$ for $1 \leq p<\infty$ equipped with the norm $\|f\|_{1}+\|f\|_{p}$ is a Segal algebra.

- Similarly, $L^{1}(G) \cap C_{0}(G)$ with respect to the norm $\|f\|_{1}+\|f\|_{\infty}$ is a Segal algebra, where $C_{0}(G)$ is the $C^{*}$-algebra of continuous functions on $G$ vanishing at infinity.

- $\quad$ Let $G$ be a compact group, $\mathcal{F}$ denote the Fourier transform, and $\mathcal{L}^{p}(\widehat{G})$ be the space which will be defined in (3.1). We can see that $\mathcal{F}^{-1}\left(\mathcal{L}^{p}(\widehat{G})\right)$, which we 
denote by $\mathfrak{C}^{p}(G)$, equipped with convolution, is a subalgebra of $L^{1}(G)$. For $\|f\|_{\mathbb{C}^{p}(G)}:=\|\mathcal{F} f\|_{\mathcal{L}^{p}(\widehat{G})}$, one can show that for each $1 \leq p \leq 2,\left(\mathbb{C}^{p}(G),\|\cdot\|_{\mathbb{C}^{p}(G)}\right)$ is a Segal algebra on $G$.

Proposition 2.2. Let $\mathcal{A}$ be a commutative semisimple regular Banach algebra and let $\mathcal{B}$ be an abstract Segal algebra of $\mathcal{A}$. Then $\mathcal{B}$ is also semisimple and regular. Moreover, $\mathcal{B}$ contains all elements $a \in \mathcal{A}$ such that $\operatorname{supp}(\hat{a})$ is compact.

Proof. By [2, Theorem 2.1], $\sigma(\mathcal{B})$ is homeomorphic to $\sigma(\mathcal{A})$ and $\mathcal{B}$ is semisimple.

Theorem 3.6.15 and Theorem 3.7.1 of [15] imply that for a commutative regular Banach algebra $\mathcal{A}$ and a closed subset $E$ of $\sigma(\mathcal{A})$ in the Gelfand spectrum topology, if $a \in \mathcal{A}$ such that $|\phi(a)| \geq \delta>0$ for every $\phi \in E$, then there exists some $a^{\prime} \in \mathcal{A}$ such that $\phi\left(a a^{\prime}\right)=1$ for every $\phi \in E$. We call this property local invertibility.

Now, [14, Proposition 2.1.14] says that if $\mathcal{A}$ is a commutative semisimple algebra with local invertibility and $\mathcal{I}$ an ideal of $\mathcal{A}$ such that $\bigcap_{a \in \mathcal{I}} \operatorname{Ker} \hat{a}=\emptyset$, then $\mathcal{I}$ contains all elements $a \in \mathcal{A}$ such that $\hat{a}$ has compact support in $\sigma(\mathcal{A})$. In particular, this applies to $\mathcal{B}$ because of density.

Since $\mathcal{A}$ is regular, for a closed set $E \subseteq \sigma(\mathcal{A})$ and $\phi \in \sigma(\mathcal{A}) \backslash E$, we have some $a \in \mathcal{A}$ such that $\left.\hat{a}\right|_{E} \equiv 0, \hat{a}(\phi)=1$, and $\operatorname{supp}(\hat{a})$ is a compact subset of $\sigma(\mathcal{A})$. Therefore, $a \in \mathcal{B}$, implying that $\mathcal{B}$ is regular.

In [3], a nice criterion is developed to prove the nonapproximate amenability of Banach algebras. At several points we will rely crucially on this criterion. For this reason, we present a version of the criterion below. Recall that for a Banach algebra $\mathcal{A}$, a sequence $\left(a_{n}\right)_{n \in \mathbb{N}} \subseteq \mathcal{A}$ is called multiplier-bounded if, for some $M>0$, $\sup _{n \in \mathbb{N}}\left\|a_{n} b\right\| \leq M\|b\|$ for all $b \in \mathcal{A}$.

Theorem 2.3. Let $\mathcal{A}$ be a Banach algebra. Suppose that there exists an unbounded but multiplier-bounded sequence $\left(a_{n}\right)_{n \geq 1} \subseteq \mathcal{A}$ such that

$$
a_{n} a_{n+1}=a_{n}=a_{n+1} a_{n}
$$

for all $n$. Then $\mathcal{A}$ is not approximately amenable.

The following theorem is the main theorem of this section.

THeORem 2.4. Let $G$ be a locally compact amenable group and $\mathrm{SA}(G)$ a proper abstract Segal algebra of $A(G)$. Then $\mathrm{SA}(G)$ is not approximately amenable.

Proof. Since $\mathrm{SA}(G)$ is a proper abstract Segal algebra of $A(G)$, the norms $\|\cdot\|_{\mathrm{SA}(G)}$ and $\|\cdot\|_{A(G)}$ can not be equivalent. On the other hand, $\|f\|_{A(G)} \leq M\|f\|_{\mathrm{SA}(G)}$ for $f \in \operatorname{SA}(G)$ and some $M>0$. Therefore, we can find a sequence $\left(f_{n}\right)_{n \in \mathbb{N}}$ in $C_{c}(G) \cap A(G)$, and hence by Proposition 2.2 in $\operatorname{SA}(G)$, such that

$$
n\left\|f_{n}\right\|_{A(G)} \leq\left\|f_{n}\right\|_{\mathrm{SA}(G)} \quad \text { for all } n \in \mathbb{N} .
$$

If $G$ is a locally compact group, then $G$ is amenable if and only if it satisfies the Leptin condition, that is, for every $\epsilon>0$ and compact set $K \subseteq G$, there exists a relatively compact neighborhood $V$ of $e$ such that $\lambda(K V) / \lambda(V)<1+\epsilon[13$, Section 2.7]. 
Fix $D>1$. Using the Leptin condition and Lemma 2.1, we can generate a sequence $\left(u_{n}\right)_{n \in \mathbb{N}}$ inductively in $A(G) \cap C_{c}(G) \subseteq \mathrm{SA}(G)$ such that $\left.u_{n}\right|_{\text {supp } f_{n}} \equiv 1$, supp $u_{n} \subseteq\{x \in G$ : $\left.u_{n+1}(x)=1\right\}$, and $\left\|u_{n}\right\|_{A(G)} \leq D$. Hence $u_{n} f_{n}=f_{n}$ and $u_{n} u_{n+1}=u_{n}$ for every $n \in \mathbb{N}$.

So here we only need to prove unboundedness of $\left(u_{n}\right)_{n \geq 1}$ in $\|\cdot\|_{\mathrm{SA}(G)}$. Suppose otherwise that $\sup _{n \in \mathbb{N}}\left\|u_{n}\right\|_{\mathrm{SA}(G)}=C^{\prime}$ for some $0<C^{\prime}<\infty$. Then for each $n \in \mathbb{N}$, one can write

$$
\left\|f_{n}\right\|_{\mathrm{SA}(G)}=\left\|u_{n} f_{n}\right\|_{\mathrm{SA}(G)} \leq C\left\|f_{n}\right\|_{A(G)}\left\|u_{n}\right\|_{\mathrm{SA}(G)} \leq C^{\prime} C\left\|f_{n}\right\|_{A(G)}
$$

for some fixed $C>0$. But this violates the condition (2.1); therefore, $\left(u_{n}\right)_{n \in \mathbb{N}}$ is unbounded in $\|\cdot\|_{\mathrm{SA}(G)}$. Consequently, Theorem 2.3 shows that $\mathrm{SA}(G)$ is not approximately amenable.

We should recall that the Leptin condition played a crucial role in the proof. As we mentioned before, the approximate amenability of all proper Segal algebras on $\mathbb{R}^{d}$ has been studied by Choi and Ghahramani in [3]. We are therefore motivated to conclude a generalization of their result in the following corollary.

Corollary 2.5. Let $G$ be a locally compact abelian group. Then every proper Segal subalgebra of $L^{1}(G)$ is not approximately amenable.

Proof. Let $S^{1}(G)$ be a proper Segal algebra on $G$. Applying the Fourier transform on $L^{1}(G)$, we may transform $S^{1}(G)$ to a proper abstract Segal algebra $\operatorname{SA}(\widehat{G})$ of $A(\widehat{G})$, the Fourier algebra on the dual of group $G$. By Theorem $2.4, \operatorname{SA}(\widehat{G})$ is not approximately amenable.

REMARK. In particular, by Theorem 2.4, for an amenable locally compact group $G$ the Lebesgue-Fourier algebra with pointwise multiplication is approximately amenable if and only if $G$ is a compact group, in which case it equals the Fourier algebra of $G$. Moreover, by Corollary 2.5, for a locally compact abelian group $G$, the LebesgueFourier algebra with convolution product is approximately amenable if and only if $G$ is discrete, in which case it equals $\ell^{1}(G)$.

\section{Hypergroups and their Fourier algebra}

Although the dual of a compact group is not a group, in general, it is a (commutative discrete) hypergroup. We give the background needed for this result in Section 3.1. Muruganandam, in [12], gave a definition of the Fourier space, $A(H)$, of a hypergroup $H$ and showed that $A(H)$ is a Banach algebra with pointwise product for certain commutative hypergroups. In Section 3.2, we study the Fourier space on the dual of a compact group $G$, denoted by $A(\widehat{G})$. We show that indeed for each compact group $G, A(\widehat{G})$ is a Banach algebra.

3.1. Preliminaries and notations. For studying hypergroups, we mainly rely on [1]. As a short summary for hypergroups we give the following definitions and facts. 
Let $(H, *, \sim)$ be a (locally compact) hypergroup possessing a Haar measure $h$. The notation $A * B$ stands for

$$
\bigcup\left\{\operatorname{supp}\left(\delta_{x} * \delta_{y}\right): \text { for all } x \in A, y \in B\right\}
$$

for $A, B$ subsets of the hypergroup $H$. With abuse of notation, we use $x * A$ to imply $\{x\} * A$. Let $C_{c}(H)$ be the space of all complex-valued, compactly supported, continuous functions over $H$. We define

$$
L_{x} f(y)=\int_{H} f(t) d \delta_{x} * \delta_{y}(t) \quad f \in C_{c}(H), x, y \in H .
$$

Defining

$$
f *_{h} g(x):=\int_{H} f(t) L_{\tilde{t}} g(x) d h(t), \quad \tilde{f}(x):=f(\tilde{x}) \quad \text { and } \quad f^{*}:=\overline{(\tilde{f}),}
$$

we can see that all of the functions $f: H \rightarrow \mathbb{C}$ such that

$$
\|f\|_{L^{1}(H, h)}:=\int_{H}|f(t)| d h(t)<\infty
$$

form a Banach $*$-algebra, denoted by $\left(L^{1}(H, h), *_{h},\|\cdot\|_{h}\right)$; it is called the hypergroup algebra of $H$.

If $H$ is discrete and $h(e)=1$,

$$
h(x)=\left(\delta_{\tilde{x}} * \delta_{x}(e)\right)^{-1} .
$$

Lemma 3.1. Let $H$ be a discrete hypergroup. For each pair $x, y \in H$,

$$
\delta_{x} *_{h} \delta_{y}(z)=\delta_{x} * \delta_{y}(z) \frac{h(x) h(y)}{h(z)}
$$

for each $z \in H$.

In this section, let $G$ be a compact group and $\widehat{G}$ the set of all irreducible unitary representations of $G$. In this paper, we follow the notation of [5] for the dual of compact groups. Where $\mathcal{H}_{\pi}$ is the finite-dimensional Hilbert space related to the representation $\pi \in \widehat{G}$, we define $\chi_{\pi}:=\operatorname{Tr} \pi$, the group character generated by $\pi$, and $d_{\pi}$ denotes the dimension of $\mathcal{H}_{\pi}$. Let $\phi=\left\{\phi_{\pi}: \pi \in \widehat{G}\right\}$ if $\phi_{\pi} \in \mathcal{B}\left(\mathcal{H}_{\pi}\right)$ for each $\pi$, and define

$$
\|\phi\|_{\mathcal{L}^{\infty}(\widehat{G})}:=\sup _{\pi}\left\|\phi_{\pi}\right\|_{\infty}
$$

for $\|\cdot\|_{\infty}$, the operator norm. The set of all those $\phi$ with $\|\phi\|_{\mathcal{L}^{\infty}(\widehat{G})}<\infty$ forms a $C^{*}$ algebra; we denote it by $\mathcal{L}^{\infty}(\widehat{G})$. It is well known that $\mathcal{L}^{\infty}(\widehat{G})$ is isomorphic to the von Neumann algebra of $G$ that is the dual of $A(G)$, see [5, 8.4.17]. We define

$$
\mathcal{L}^{p}(\widehat{G})=\left\{\phi \in \mathcal{L}^{\infty}(\widehat{G}):\|\phi\|_{\mathcal{L}^{p}(\widehat{G})}^{p}:=\sum_{\pi \in \widehat{G}} d_{\pi}\left\|\phi_{\pi}\right\|_{p}^{p}<\infty\right\},
$$


for $\|\cdot\|_{p}$, the $p$-Schatten norm. For each $p, \mathcal{L}^{p}(\widehat{G})$ is an ideal of $\mathcal{L}^{\infty}(\widehat{G})$, see [5, Section 8.3]. Moreover, we define

$$
C_{0}(\widehat{G})=\left\{\phi \in \mathcal{L}^{\infty}(\widehat{G}): \lim _{\pi \rightarrow \infty}\left\|\phi_{\pi}\right\|_{\infty}=0\right\} .
$$

For each $f \in L^{1}(G), \mathcal{F}(f)=(\hat{f}(\pi))_{\pi \in \widehat{G}}$ belongs to $C_{0}(\widehat{G})$, where $\mathcal{F}$ denotes the Fourier transform and

$$
\hat{f}(\pi)=\int_{G} f(x) \pi\left(x^{-1}\right) d x .
$$

Indeed, $\mathcal{F}\left(L^{1}(G)\right)$ is a dense subset of $C_{0}(\widehat{G})$ and $\mathcal{F}$ is an isomorphism from the Banach algebra $L^{1}(G)$ onto its image.

For each two irreducible representations $\pi_{1}, \pi_{2} \in \widehat{G}$, we know that $\pi_{1} \otimes \pi_{2}$ can be written as a direct product of $\pi_{1}^{\prime}, \ldots, \pi_{n}^{\prime}$ elements of $\widehat{G}$ with respective multiplicities $m_{1}, \ldots, m_{n}$, that is,

$$
\pi_{1} \otimes \pi_{2} \cong \bigoplus_{i=1}^{n} m_{i} \pi_{i}^{\prime}
$$

We define a convolution on $\ell^{1}(\widehat{G})$ by

$$
\delta_{\pi_{1}} * \delta_{\pi_{2}}:=\sum_{i=1}^{n} \frac{m_{i} d_{\pi_{i}^{\prime}}}{d_{\pi_{1}} d_{\pi_{2}}} \delta_{\pi_{i}^{\prime}}
$$

and define an involution by $\tilde{\pi}=\bar{\pi}$ for all $\pi, \pi_{1}, \pi_{2} \in \widehat{G}$. It is straightforward to verify that $(\widehat{G}, *, \sim)$ forms a discrete commutative hypergroup such that $\pi_{0}$, the trivial representation of $G$, is the identity element of $\widehat{G}$ and $h(\pi)=d_{\pi}^{2}$ is the Haar measure of $\widehat{G}$.

EXAmple 3.2. Let $\widehat{S U(2)}$ be the hypergroup of all irreducible representations of the compact group $\mathrm{SU}(2)$. We know that

$$
\widehat{\mathrm{SU}(2)}=\left(\pi_{\ell}\right)_{\ell \in 0, \frac{1}{2}, 1, \frac{3}{2}, \ldots},
$$

where the dimension of $\pi_{\ell}$ is $2 \ell+1$, see [9, 29.13]. Moreover,

$$
\pi_{\ell} \oplus \pi_{\ell^{\prime}}=\bigoplus_{r=\left|\ell-\ell^{\prime}\right|}^{\ell+\ell^{\prime}} \pi_{r}=\pi_{\left|\ell-\ell^{\prime}\right|} \oplus \pi_{\left|\ell-\ell^{\prime}\right|+1} \oplus \cdots \oplus \pi_{\ell+\ell^{\prime}} \quad \text { (by [9, Theorem 29.26]). }
$$

So, using definition (3.2), we have that

$$
\delta_{\pi_{\ell}} * \delta_{\pi_{\ell^{\prime}}}=\sum_{r=\left|\ell-\ell^{\prime}\right|}^{\ell+\ell^{\prime}} \frac{(2 r+1)}{(2 \ell+1)\left(2 \ell^{\prime}+1\right)} \delta_{\pi_{r}} .
$$

Also $\tilde{\pi}_{\ell}=\pi_{\ell}$ and $h\left(\pi_{\ell}\right)=(2 \ell+1)^{2}$ for all $\ell$. 
Example 3.3. Suppose that $\left\{G_{i}\right\}_{i \in \mathbf{I}}$ is a nonempty family of compact groups for arbitrary indexing set $\mathbf{I}$. Let $G:=\prod_{i \in \mathbf{I}} G_{i}$ be the product of $\left\{G_{i}\right\}_{i \in \mathbf{I}}$, that is, $G=\left\{\left(x_{i}\right)_{i \in \mathbf{I}}\right.$ : $x_{i} \in G_{i}$ \} equipped with product topology. Then $G$ is a compact group and by [9, Theorem 27.43],

$$
\widehat{G}=\left\{\pi=\bigotimes_{i \in \mathbf{I}} \pi_{i}: \text { such that } \pi_{i} \in \widehat{G}_{i} \text { and } \pi_{i}=\pi_{0} \text { except for finitely many } i\right\}
$$

equipped with the discrete topology. Moreover, for each $\pi=\bigotimes_{i \in \mathbf{I}} \pi_{i} \in \widehat{G}, d_{\pi}=$ $\prod_{i \in \mathbf{I}} d_{\pi_{i}}$.

If $\pi_{k}=\bigotimes_{i \in \mathbf{I}} \pi_{i}^{(k)} \in \widehat{G}$ for $k=1,2$, one can show that

$$
\delta_{\pi_{1}} * \delta_{\pi_{2}}(\pi)=\prod_{i \in \mathbf{I}} \delta_{\pi_{i}^{(1)}} * \widehat{G}_{i} \delta_{\pi_{i}^{(2)}}\left(\pi_{i}\right) \quad \text { for } \pi=\bigotimes_{i \in \mathbf{I}} \pi_{i} \in \widehat{G},
$$

where $* \widehat{G}_{i}$ is the hypergroup product in $\widehat{G}_{i}$ for each $i \in \mathbf{I}$. Also, each character $\chi$ of $G$ corresponds to a family of characters $\left(\chi_{i}\right)_{i \in \mathbf{I}}$ such that $\chi_{i}$ is a character of $G_{i}$ and $\chi(x)=\prod_{i \in \mathbf{I}} \chi_{i}\left(x_{i}\right)$ for each $x=\left(x_{i}\right)_{i \in \mathbf{I}} \in G$. Note that $\chi_{i} \equiv 1$ for all of $i \in \mathbf{I}$ except finitely many.

3.2. The Fourier algebra of the dual of a compact group. For a compact hypergroup $H$, Vrem in [17] defined the Fourier space similar to the Fourier algebra of a compact group. Later, Muruganandam, in [12], defined the Fourier-Stieltjes space on an arbitrary (not necessarily compact) hypergroup $H$ using irreducible representations of $H$ analogous to the Fourier-Stieltjes algebra on locally compact groups. Subsequently, he defined the Fourier space of a hypergroup $H$, as a closed subspace of the Fourier-Stieltjes algebra, generated by $\left\{f *_{h} \tilde{f}: f \in L^{2}(H, h)\right\}$. Muruganandam also showed that when $H$ is commutative, $A(H)$ is $\left\{f *_{h} \tilde{g}: f, g \in\right.$ $\left.L^{2}(H, h)\right\}$ and $\|u\|_{A(H)}=\inf \|f\|_{2}\|g\|_{2}$ for all $f, g \in L^{2}(H, h)$ such that $u=f * \tilde{g}$. He calls the hypergroup $H$ a regular Fourier hypergroup, if the Banach space $\left(A(H),\|\cdot\|_{A(H)}\right)$ equipped with pointwise product is a Banach algebra.

We prove a hypergroup version of Lemma 2.1 which shows some important properties of the Banach space $A(H)$ for an arbitrary hypergroup $H$ (not necessarily a regular Fourier hypergroup). Some parts of the following lemma have already been shown in [17] for compact hypergroups and that proof is applicable to general hypergroups. Here we present a complete proof for the lemma.

Lemma 3.4. Let $H$ be a hypergroup, $K$ a compact subset of $H$ and $U$ an open subset of $H$ such that $K \subset U$. Then for each relatively compact open set $V$ such that $\overline{K * V * \tilde{V}} \subseteq U$, there exists some $u_{V} \in A(H) \cap C_{c}(H)$ such that:
(1) $u_{V}(H) \geq 0$;
(2) $\left.u_{V}\right|_{K}=1$;
(3) $\operatorname{supp}\left(u_{V}\right) \subseteq U$;
(4) $\left\|u_{V}\right\|_{A(H)} \leq\left(h_{H}(K * V) / h_{H}(V)\right)^{\frac{1}{2}}$. 
Proof. Let us define

$$
u_{V}:=\frac{1}{h_{H}(V)} 1_{K * V} *_{h} \tilde{1}_{V} .
$$

Clearly $u_{V} \geq 0$. Moreover, for each $x \in K$,

$$
\begin{aligned}
h_{H}(V) u_{V}(x) & =1_{K * V} *_{h} \tilde{1}_{V}(x) \\
& =\int_{H} 1_{K * V}(t) L_{\tilde{t}} \tilde{1}_{V}(x) d h_{H}(t) \\
& =\int_{H} 1_{K * V}(t) L_{\tilde{x}} 1_{V}(t) d h_{H}(t) \\
& =\int_{t \in H} L_{x} 1_{K * V}(t) 1_{V}(t) d h_{H}(t) \quad \text { by [1, Theorem 1.3.21]) } \\
& =\int_{V}\left\langle 1_{K * V}, \delta_{x} * \delta_{t}\right\rangle d h_{H}(t) \\
& =h_{H}(V) .
\end{aligned}
$$

Also [1, Proposition 1.2.12] implies that

$$
\operatorname{supp}\left(1_{K * V} *_{h} \tilde{1}_{V}\right) \subseteq \overline{(K * V * \tilde{V})} \subseteq U,
$$

which implies that $u_{V}$ is compact supported. Finally, by [12, Proposition 2.8], we know that

$$
\left\|u_{V}\right\|_{A(H)} \leq \frac{\left\|1_{K * V}\right\|_{2}\left\|1_{V}\right\|_{2}}{h_{H}(V)}=\frac{h_{H}(K * V)^{\frac{1}{2}} h_{H}\left(1_{V}\right)^{\frac{1}{2}}}{h_{H}(V)}=\frac{h_{H}(K * V)^{\frac{1}{2}}}{h_{H}(V)^{\frac{1}{2}}} .
$$

This concludes the proof.

Remark. For each pair $K, U$ such that $K \subset U$, we can always find a relatively compact neighborhood $V$ of $e_{H}$ that satisfies the conditions in Lemma 3.4. But the proof is quite long and in our application the existence of such $V$ will be clear.

Given a commutative hypergroup, it is not immediate whether it is a regular Fourier hypergroup or not. We will show that when $G$ is a compact group, the hypergroup $\widehat{G}$ is a regular Fourier hypergroup.

Definition 3.6. For $A(G)$, the Fourier algebra on $G$, we define

$$
Z A(G):=\left\{f \in A(G): f\left(y x y^{-1}\right)=f(x) \text { for all } x \in G\right\},
$$

which is a Banach algebra with pointwise product and $\|\cdot\|_{A(G)}$.

THEOREM 3.7. Let $G$ be a compact group. Then $\widehat{G}$ is a regular Fourier hypergroup and $A(\widehat{G})$ is isometrically isomorphic with the center of the group algebra $G$, that is, $A(\widehat{G}) \cong Z L^{1}(G)$. Moreover, the hypergroup algebra of $\widehat{G}, L^{1}(\widehat{G}, h)$, is isometrically isomorphic with $Z A(G)$. 
Proof. Let $\mathcal{F}$ be the Fourier transform on $L^{1}(G)$. We know that $\left.\mathcal{F}\right|_{L^{2}(G)}$ is an isometric isomorphism from $L^{2}(G)$ onto $\mathcal{L}^{2}(\widehat{G})$. By the properties of the Fourier transform, [5, Proposition 4.2], for each $f \in Z L^{2}(G)$ and $g \in L^{1}(G)$

$$
\mathcal{F}(f) \circ \mathcal{F}(g)=\mathcal{F}(f * g)=\mathcal{F}(g * f)=\mathcal{F}(g) \circ \mathcal{F}(f) .
$$

So $\mathcal{F}(f)$ commutes with all elements of $C_{0}(\widehat{G})$; therefore, $\mathcal{F}(f)=\left(\alpha_{\pi} I_{d_{\pi} \times d_{\pi}}\right)_{\pi \in \widehat{G}}$ for a family of scalars $\left(\alpha_{\pi}\right)_{\pi \in \widehat{G}}$ in $\mathbb{C}$. Hence,

$$
\|\mathcal{F}(f)\|_{2}^{2}=\sum_{\pi \in \widehat{G}} d_{\pi}\|\widehat{f}(\pi)\|_{2}^{2}=\sum_{\pi \in \widehat{G}} d_{\pi} \alpha_{\pi}^{2}\left\|I_{d_{\pi} \times d_{\pi}}\right\|_{2}^{2}=\sum_{\pi \in \widehat{G}} \alpha_{\pi}^{2} d_{\pi}^{2}=\sum_{\pi \in \widehat{G}} \alpha_{\pi}^{2} h(\pi) .
$$

Using the preceding identity, we define $\mathcal{T}: Z L^{2}(G) \rightarrow L^{2}(\widehat{G}, h)$ by $\mathcal{T}(f)=\left(\alpha_{\pi}\right)_{\pi \in \widehat{G}}$. Note that $\left\{\chi_{\tilde{\pi}}\right\}_{\pi \in \widehat{G}}$ forms an orthonormal basis for $Z L^{2}(G)$. Since $\mathcal{F}\left(\chi_{\tilde{\pi}}\right)=d_{\tilde{\pi}}^{-1} I_{d_{\tilde{\pi} \times \tilde{\pi}}}$, $\mathcal{T}(\tilde{f})=\mathcal{T}\left(f \tilde{)}\right.$ for each $f \in L^{2}(G)$, where $\tilde{f}(x)=f\left(x^{-1}\right)$. So $\mathcal{T}$ is an isometric isomorphism from $Z L^{2}(G)$ onto $L^{2}(\widehat{G}, h)$.

We claim that $\mathcal{T}(f \tilde{g})=\mathcal{T}(f) *_{h} \mathcal{T}(g)$ for all $f, g \in Z L^{2}(G)$. To prove our claim it is enough to show that $\mathcal{T}\left(\chi_{\pi_{1}} \chi_{\pi_{2}}\right)=\mathcal{T}\left(\chi_{\pi_{1}}\right) *_{h} \mathcal{T}\left(\chi_{\pi_{2}}\right)$ for $\pi_{1}, \pi_{2} \in \widehat{G}$. Therefore, using Lemma 3.1, for each two representations $\pi_{1}, \pi_{2} \in \widehat{G}$,

$$
\begin{aligned}
\mathcal{T}\left(\chi_{\pi_{1}} \chi_{\pi_{2}}\right) & =\mathcal{T}\left(\sum_{i=1}^{n} m_{i} \chi_{\pi_{i}^{\prime}}\right) \\
& =\sum_{i=1}^{n} m_{i} \mathcal{T}\left(\chi_{\pi_{i}^{\prime}}\right) \\
& =\sum_{i=1}^{n} m_{i} d_{\pi_{i}^{\prime}}^{-1} \delta_{\pi_{i}^{\prime}} \\
& =d_{\pi_{1}}^{-1} \delta_{\pi_{1}} *_{h} d_{\pi_{2}}^{-1} \delta_{\pi_{2}} \\
& =\mathcal{T}\left(\chi_{\pi_{1}}\right) *{ }_{h} \mathcal{T}\left(\chi_{\pi_{2}}\right) .
\end{aligned}
$$

Now we can define a surjective extension $\mathcal{T}: Z L^{1}(G) \rightarrow A(\widehat{G})$, using the fact that $\operatorname{lin}\left\{\chi_{\pi}\right\}_{\pi \in \widehat{G}}$ is dense in $Z L^{1}(G)$ as well and $\|f\|_{1}=\inf \left\|g_{1}\right\|_{2}\left\|g_{2}\right\|_{2}$ for all $g_{1}, g_{2} \in L^{2}(G)$ such that $f=g_{1} \tilde{g_{2}}$. Using the definition of the norm of $A(\widehat{G}),\|\mathcal{T}(f)\|_{A(\widehat{G})}=\|f\|_{1}$ for each $f \in Z L^{1}(G)$. To show that the extension of $\mathcal{T}$ is onto, for each pair $g_{1}, g_{2} \in$ $Z L^{2}(G)$, we note that $g_{1} \tilde{g_{2}} \in Z L^{1}(G)$. So, $\mathcal{T}$ is an isometric isomorphism. This implies that $A(\widehat{G})$ is a Banach algebra with pointwise product and hence $\left(A(\widehat{G}), \cdot\|\cdot\|_{A(\widehat{G})}\right) \cong$ $\left(Z L^{1}(G), *,\|\cdot\|_{1}\right)$.

The second part is similar to the first part of the proof. This time we consider the restriction of the Fourier transform from $Z A(G)$ onto $\mathcal{L}^{1}(\widehat{G})$. Again by an argument similar to (3.3), we define an isometric mapping $\mathcal{T}^{\prime}$ from $Z A(G)$ onto $L^{1}(\widehat{G}, h)$. Since $\operatorname{lin}\left\{\chi_{\pi}\right\}_{\pi \in \widehat{G}}$ is dense in $Z A(G)$, we observe that $\mathcal{T}^{\prime}$ is an isometric isomorphism from $Z A(G)$ as a $*$-algebra with complex conjugate and pointwise product onto $L^{1}(\widehat{G}, h)$ as a $*$-algebra with convolution $*_{h}$. 


\section{The Leptin condition on hypergroups}

In the proof of Theorem 2.4, we used the Leptin condition for amenable groups. In this subsection we study the Leptin condition for hypergroups. In [16], the Reiter condition was introduced for amenable hypergroups. Although the Reiter condition on hypergroups behaves similar to amenable groups, the Leptin condition is a problem for hypergroups. This difficulty is resulted mainly from the following fact: in general hypergroups, when we convolve the Dirac measures of two elements, although the probabilistic measure which is yielded from this convolution is finitely supported, we do not necessarily have a bound on the cardinality of its size. There are some attempts to answer this question for some special hypergroups in [10].

Recall that for each two subsets $A$ and $B$ of $X$, we denote the set $(A \backslash B) \cup(B \backslash A)$ by $A \triangle B$.

Definition 4.1. Let $H$ be a hypergroup. We say that $H$ satisfies the Leptin condition if for every compact subset $K$ of $H$ and $\epsilon>0$, there exists a measurable set $V$ in $H$ such that $0<h(V)<\infty$ and $h(K * V) / h(V)<1+\epsilon$.

We will use the Leptin condition, in the case where $H$ is the dual of a compact group $G$, to study approximate amenability for Segal algebras on $G$.

REMARK. In the definition of the Leptin condition mentioned above, we can suppose that $V$ is a compact measurable set. To show this fact suppose that $H$ satisfies the Leptin condition. For a compact subset $K$ of $H$ and $\epsilon>0$, there exists a measurable set $V$ such that $h(K * V) / h(V)<1+\epsilon$. Using regularity of $h$, for each positive integer $n$, we can find compact set $V_{1} \subseteq V$ such that $h\left(V \backslash V_{1}\right)<h(V) / n$. This implies that $0<h\left(V_{1}\right)$ and $h(V) / h\left(V_{1}\right)<n /(n-1)$. Therefore,

$$
\frac{h\left(K * V_{1}\right)}{h\left(V_{1}\right)} \leq \frac{h(V)}{h\left(V_{1}\right)}\left(\frac{h\left(K * V_{1}\right)}{h(V)}\right)<\frac{n}{n-1}(1+\epsilon) .
$$

So we can add compactness of $V$ to the definition of the Leptin condition.

Note that since the duals of compact groups are commutative, they are all amenable hypergroups [16], but as we mentioned this amenability does not say anything about the Leptin condition on these hypergroups. So the next question is: for which compact groups $G$ do the hypergroups $\widehat{G}$ satisfy the Leptin condition? We will now show that Examples 3.2 and 3.3 sometimes satisfy this condition.

Proposition 4.3. The hypergroup $\widehat{\mathrm{SU}(2)}$ satisfies the Leptin condition.

Proof. Take a compact subset $K$ of $\widehat{\mathrm{SU}(2)}$ and $\epsilon>0, k:=\sup \left\{\ell: \pi_{\ell} \in K\right\}$. We select $m \geq k$ such that for $V=\left\{\pi_{\ell}\right\}_{\ell=0}^{m}$,

$$
\begin{aligned}
\frac{h\left(\pi_{k} * V\right)}{h(V)} & =\frac{\sum_{\ell=1}^{2 m+2 k+1} \ell^{2}}{\sum_{\ell=1}^{2 m+1} \ell^{2}} \\
& =\frac{\frac{1}{3}(2 m+2 k+1)^{3}+\frac{1}{2}(2 m+2 k+1)^{2}+\frac{1}{6}(2 m+2 k+1)}{\frac{1}{3}(2 m+1)^{3}+\frac{1}{2}(2 m+1)^{2}+\frac{1}{6}(2 m+1)}<1+\epsilon .
\end{aligned}
$$


Then for each $x \in K, x * V \subseteq \pi_{k} * V$. So, using (4.1),

$$
\frac{h(K * V)}{h(V)}=\frac{h\left(\pi_{k} * V\right)}{h(V)}<1+\epsilon .
$$

This concludes the proof.

Proposition 4.4. Let $\left\{G_{i}\right\}_{i \in \mathbf{I}}$ be a family of compact groups whose duals have the Leptin condition and $G=\prod_{i \in \mathbf{I}} G_{i}$ be their product equipped with product topology. Then $\widehat{G}$ satisfies the Leptin condition.

Proof. Let $K \subseteq \widehat{G}$ be an arbitrary compact subset. Then, there exists some $F \subseteq \mathbf{I}$ finite such that $K \subseteq \bigotimes_{i \in F} K_{i} \otimes E_{F}^{c}$, where $K_{i}$ is a compact subset of $\widehat{G}_{i}$, and $E_{F}^{c}=\bigotimes_{i \in \mathbf{I} \backslash F} \pi_{0}$, where the $\pi_{0}$ are the trivial representations of the corresponding $\widehat{G}_{i}$.

Using the Leptin condition for each $G_{i}$, there exists some compact set $V_{i}$ which satisfies the Leptin condition for $K_{i}$ and $\epsilon>0$, that is, $h_{G_{i}}\left(K_{i} * V_{i}\right)<(1+\epsilon) h_{G_{i}}\left(V_{i}\right)$. Therefore, for the compact set $V=\left(\bigotimes_{i \in F} V_{i}\right) \otimes E_{F}^{c}$,

$$
\frac{h(K * V)}{h(V)} \leq \prod_{i \in F} \frac{h_{G_{i}}\left(K_{i} * V_{i}\right)}{h_{G_{i}}\left(V_{i}\right)}<(1+\epsilon)^{|F|} .
$$

This concludes the proof.

REMARK. If $G$ is finite then $\widehat{G}$ satisfies the Leptin condition; hence, for a family of finite groups, say $\left\{G_{i}\right\}_{i \in \mathbf{I}}, G:=\prod_{i \in \mathbf{I}} G_{i}, \widehat{G}$ satisfies the Leptin condition.

\section{Segal algebras on compact groups whose duals satisfy the Leptin condition}

In this section, we apply this hypergroup approach to answer the question of approximate amenability of Segal algebras. We show that every proper Segal algebra on $G$ is not approximately amenable if $G$ is a compact group and $\widehat{G}$ satisfies the Leptin condition. To prove this theorem we need some preliminary results. The proof of the following lemma is adopted from [11, Lemma 1].

Lemma 5.1. Let $\mathcal{A}$ be a Banach algebra and $\mathcal{J}$ be a dense left ideal of $\mathcal{A}$. Then for each idempotent element $p$ in the center of the algebra $\mathcal{A}$, that is, $p^{2}=p \in Z(\mathcal{A}), p$ belongs to $\mathcal{J}$.

Proof. Since $\mathcal{J}$ is dense in $\mathcal{A}$, there exists an element $a \in \mathcal{J}$ such that $\|p-a\|_{\mathcal{A}}<1$. Let us define

$$
b:=p+\sum_{n=1}^{\infty}(p-a)^{n}
$$


One can check that $p b-p b(p-a)=p b a$, which is an element in $\mathcal{J}$. On the other hand,

$$
\begin{aligned}
p b-p b(p-a) & =p\left(p+\sum_{n=1}^{\infty}(p-a)^{n}\right)-p\left(p+\sum_{n=1}^{\infty}(p-a)^{n}\right)(p-a) \\
& =p+p \sum_{n=1}^{\infty}(p-a)^{n}-p \sum_{n=2}^{\infty}(p-a)^{n}-p(p-a) \\
& =p+p(p-a)-p(p-a)=p .
\end{aligned}
$$

This concludes the proof.

Corollary 5.2. Let $G$ be a compact group. Then $\operatorname{lin}\left\{\chi_{\pi}\right\}_{\pi \in \widehat{G}}$ is $\|\cdot\|_{1}$-dense in $Z L^{1}(G)$ and for every Segal algebra $S^{1}(G)$, its center, $Z S^{1}(G)$, contains $\operatorname{lin}\left\{\chi_{\pi}\right\}_{\pi \in \widehat{G}}$.

Proof. Let $\mathcal{T}$ be the map defined in the proof of Theorem 3.7. Then, $\mathcal{T}\left(Z L^{1}(G)\right)=$ $A(\widehat{G})$. Also for the discrete hypergroup $\widehat{G}, A(\widehat{G})$ equals the $\|\cdot\|_{A(\widehat{G})}$-closure of $\operatorname{lin}\left\{\delta_{\pi}\right\}_{\pi \in \widehat{G}}$ [12]. Therefore, $Z L^{1}(G)$ is the $\|\cdot\|_{1}$-closure of $\operatorname{lin}\left\{\chi_{\pi}\right\}_{\pi \in \widehat{G}}$, since $\mathcal{T}\left(\chi_{\pi}\right)=$ $d_{\pi}^{-1} \delta_{\pi}$ for each $\pi \in \widehat{G}$. On the other hand, by Lemma 5.1, $S^{1}(G)$ contains all central idempotents $d_{\pi} \chi_{\pi}$ for each $\pi \in \widehat{G}$.

The main theorem of this section is as follows.

Theorem 5.3. Let $G$ be a compact group such that $\widehat{G}$ satisfies the Leptin condition. Then every proper Segal algebra on $G$ is not approximately amenable.

Proof. Let $S^{1}(G)$ be a proper Segal algebra on $G$. Fix $D>1$. Using the Leptin condition on $\widehat{G}$, for every arbitrary nonvoid finite set $K$ in $\widehat{G}$, we can find a finite subset $V_{K}$ of $\widehat{G}$ such that $h\left(K * V_{K}\right) / h\left(V_{K}\right)<D^{2}$. Using Lemma 3.4, for

$$
v_{K}:=\frac{1}{h\left(V_{K}\right)} 1_{K * V_{K}} *_{h} \tilde{1}_{V_{K}}
$$

we have $\left\|v_{K}\right\|_{A(\widehat{G})}<D$ and $\left.v_{K}\right|_{K} \equiv 1$. We consider the net $\left\{v_{K}: K \subseteq \widehat{G}\right.$ compact $\}$ in $A(\widehat{G})$ where $v_{K_{2}} \geq v_{K_{1}}$ whenever $v_{K_{1}} v_{K_{2}}=v_{K_{1}}$. So $\left(v_{K}\right)_{K \subseteq \widehat{G}}$ forms a $\|\cdot\|_{A(\widehat{G})}$-bounded net in $A(\widehat{G}) \cap c_{c}(\widehat{G})$. Let $f \in A(\widehat{G}) \cap c_{c}(\widehat{G})$ with $K=\operatorname{supp} f$. Then $v_{K} f=f$. Therefore, $\left(v_{K}\right)_{K \subseteq \widehat{G}}$ is a bounded approximate identity of $A(\widehat{G})$.

Using $\mathcal{T}$ defined in the proof of Theorem 3.7, we can define the net $\left(u_{K}\right)_{K \subseteq \widehat{G}}$ in $S^{1}(G)$ by $u_{K}:=\mathcal{T}^{-1}\left(v_{K}\right)$. We show that $\left(u_{K}\right)_{K \subseteq \widehat{G}}$ satisfies some conditions. First of all, since $\mathcal{T}$ is an isometry from $Z L^{1}(G)$ onto $A(\widehat{G}),\left(u_{K}\right)_{K \subseteq \widehat{G}}$ is a $\|\cdot\|_{1}$-bounded net in $S^{1}(G)$, by Lemma 5.1. Moreover, since $\mathcal{T}$ is an isomorphism,

$$
u_{K_{1}} * u_{K_{2}}=\mathcal{T}^{-1}\left(v_{K_{1}}\right) * \mathcal{T}^{-1}\left(v_{K_{2}}\right)=\mathcal{T}^{-1}\left(v_{K_{1}} v_{K_{2}}\right)=\mathcal{T}^{-1}\left(v_{K_{1}}\right)=u_{K_{1}}
$$

for $v_{K_{2}} \geq v_{K_{1}}$, which we equivalently denote by $u_{K_{2}} \geq u_{K_{1}}$. Let $\left(u_{K}\right)_{K \subseteq \widehat{G}}$ be the net constructed. 
Claim. For every $K_{0} \subseteq \widehat{G}, K_{0}$ finite, the net $\left\{u_{K}: u_{K} \geq u_{K_{0}}\right\}$ is unbounded in the norm of $S^{1}(G)$.

To prove the claim, assume towards a contradiction that there exists a finite $K_{0}$ and $C>0$ such that $\left\|u_{K}\right\|_{S^{1}(G)} \leq C$ for all $u_{K} \geq u_{K_{0}}$. Since $G$ is compact and $S^{1}(G)$ is a Segal algebra, we know, by [11], that $S^{1}(G)$ has a central approximate identity which is bounded in $L^{1}$-norm. Denote this by $\left(e_{\alpha}\right)_{\alpha}$. By Corollary 5.2, let us generate a net $\left(e_{\alpha, \epsilon}^{\prime}\right)_{\alpha, 1>\epsilon>0}$ in $\operatorname{lin}\left\{\chi_{\pi}\right\}_{\pi \in \widehat{G}}$, where $\left\|e_{\alpha, \epsilon}^{\prime}-e_{\alpha}\right\|_{1}<\epsilon$ for each pair of $(\alpha, \epsilon)$; therefore, $\left(e_{\alpha, \epsilon}^{\prime}\right)_{\alpha, 1>\epsilon>0} \subseteq Z S^{1}(G)$. We show that $\left(e_{\alpha, \epsilon}^{\prime}\right)_{\alpha, 1>\epsilon>0}$ is still a central $\|\cdot\|_{1}$-bounded approximate identity of $S^{1}(G)$. Doing so, for each $f \in S^{1}(G)$, note that

$$
\begin{aligned}
\left\|e_{\alpha, \epsilon}^{\prime} * f-f\right\|_{S^{1}(G)} & \leq\left\|e_{\alpha, \epsilon}^{\prime} * f-e_{\alpha} * f\right\|_{S^{1}(G)}+\left\|e_{\alpha} * f-f\right\|_{S^{1}(G)} \\
& \leq\left\|e_{\alpha, \epsilon}^{\prime}-e_{\alpha}\right\|_{1}\|f\|_{S^{1}(G)}+\left\|e_{\alpha} * f-f\right\|_{S^{1}(G)},
\end{aligned}
$$

which goes toward 0 as $\alpha$ grows and $\epsilon \rightarrow 0$. Moreover, for each $(\alpha, \epsilon)$, there exists some finite set $K^{\prime}$ such that $K_{0} \subseteq K^{\prime} \subseteq \widehat{G}$ and $\mathcal{T}\left(e_{\alpha, \epsilon}^{\prime}\right) v_{K}=\mathcal{T}\left(e_{\alpha, \epsilon}^{\prime}\right)$ for each $v_{K^{\prime}} \preccurlyeq v_{K}$; hence,

$$
\left\|e_{\alpha, \epsilon}^{\prime}\right\|_{S^{1}(G)}=\lim _{K_{0} \subseteq K \rightarrow \widehat{G}}\left\|e_{\alpha, \epsilon}^{\prime} * u_{K}\right\|_{S^{1}(G)} .
$$

Consequently,

$$
\begin{aligned}
\left\|e_{\alpha, \epsilon}^{\prime}\right\|_{S^{1}(G)} & =\lim _{K_{0} \subseteq K \rightarrow \widehat{G}}\left\|e_{\alpha, \epsilon}^{\prime} * u_{K}\right\|_{S^{1}(G)} \\
& \leq \sup _{K_{0} \subset K \subseteq \widehat{G}}\left\|e_{\alpha, \epsilon}^{\prime}\right\|\left\|_{1}\right\| u_{K}\left\|_{S^{1}(G)} \leq C\right\| e_{\alpha, \epsilon}^{\prime} \|_{1} .
\end{aligned}
$$

This implies that $\left(e_{\alpha, \epsilon}^{\prime}\right)_{\alpha, 1>\epsilon>0}$ is $\|\cdot\|_{S^{1}(G)}$-bounded. But a Segal algebra cannot have a bounded approximate identity unless it coincides with the group algebra [2], which contradicts the properness of $S^{1}(G)$. Hence, the claim is proved.

To generate a sequence which satisfies the conditions of Theorem 2.3, fix a nonempty finite set $K_{0} \subseteq \widehat{G}$. By our claim, we inductively construct a sequence of finite sets $K_{0} \subset K_{1} \subset \cdots$ in $\widehat{G}$ such that $u_{K_{n}} \geq u_{K_{n-1}}$ and $\left\|u_{K_{n}}\right\|_{S^{1}(G)} \geq n$ for all $n \in \mathbb{N}$. Since $u_{K_{n}} * u_{K_{n-1}}=u_{K_{n-1}}$, by Theorem $2.3, S^{1}(G)$ is not approximately amenable.

Corollary 5.4. Every proper Segal algebra on $\mathrm{SU}(2)$ is not approximately amenable.

Remark. After this work was submitted, we found that a weaker version of the Leptin condition is satisfied by the dual of SU(3). One can use this to show that proper Segal algebras on SU(3) are not approximately amenable. This will appear in a subsequent paper which is currently in preparation.

COROLlaRy 5.6. Let $\left\{G_{i}\right\}_{i \in \mathbf{I}}$ be a nonempty family of compact groups whose duals satisfy the Leptin condition, and $G=\prod_{i \in \mathbf{I}} G_{i}$ equipped with product topology. Then every proper Segal algebra on $G$ is not approximately amenable. 


\section{Further questions}

- Is every proper Segal algebra on a compact group $G$ not approximately amenable? This question was first asked about locally compact abelian groups by Dales and Loy [4], which we have answered in this paper. We have also shown that the conjecture holds for the compact group SU(2) and for products of finite groups.

- For which other compact groups do their duals satisfy the Leptin condition? Can one find a compact group $G$ whose dual does not satisfy the Leptin condition? One may want to study the Leptin condition for the dual of other compact connected Lie groups, say SU(n). A further study on SU(3), done by the author, suggests that $\mathrm{SU(3)}$ may not satisfy the Leptin condition. But this statement is still a conjecture.

- For locally compact groups, the existence of a bounded approximate identity of the Fourier algebra implies the Leptin condition. In the hypergroup case, it seems that we cannot prove this implication. Can we find a regular Fourier hypergroup $H$ whose Fourier algebra has a bounded approximate identity while $H$ does not satisfies the Leptin condition?

\section{Acknowledgements}

The author would like to express his deep gratitude to Yemon Choi and Ebrahim Samei, his supervisors, for their kind help and constant encouragement. The author also thanks the referee for his/her comments and recommendations.

\section{References}

[1] W. R. Bloom and H. Heyer, Harmonic Analysis of Probability Measures on Hypergroups, de Gruyter Studies in Mathematics, 20 (Walter de Gruyter, Berlin, 1995).

[2] J. T. Burnham, 'Closed ideals in subalgebras of Banach algebras. I', Proc. Amer. Math. Soc. 32 (1972), 551-555.

[3] Y. Choi and F. Ghahramani, 'Approximate amenability of Schatten classes, Lipschitz algebras and second duals of Fourier algebras', Q. J. Math. 62 (2011), 39-58.

[4] H. G. Dales and R. J. Loy, 'Approximate amenability of semigroup algebras and Segal algebras', Dissertationes Math. (Rozprawy Mat.) 474 (2010), 58 pages.

[5] C. F. Dunkl and D. E. Ramirez, Topics in Harmonic Analysis, Appleton-Century Mathematics Series (Appleton-Century-Crofts, New York, 1971).

[6] P. Eymard, 'L'algébre de Fourier d'un groupe localement compact', Bull. Soc. Math. France 92 (1964), 181-236.

[7] F. Ghahramani and A. T. M. Lau, 'Weak amenability of certain classes of Banach algebras without bounded approximate identities', Math. Proc. Cambridge Philos. Soc. 133 (2002), 357-371.

[8] F. Ghahramani and R. J. Loy, 'Generalized notions of amenability', J. Funct. Anal. 208(1) (2004), 229-260.

[9] E. Hewitt and K. A. Ross, Abstract Harmonic Analysis. Vol. II: Structure and Analysis for Compact Groups. Analysis on Locally Compact Abelian Groups, Die Grundlehren der mathematischen Wissenschaften, 152 (Springer, New York-Berlin, 1970).

[10] V. Hösel, M. Hofmann and R. Lasser, 'Means and Følner condition on polynomial hypergroups', Mediterr. J. Math. 7(1) (2010), 75-88. 
[11] E. Kotzmann and H. Rindler, 'Segal algebras on nonabelian groups', Trans. Amer. Math. Soc. 237 (1978), 271-281.

[12] V. Muruganandam, 'Fourier algebra of a hypergroup. I', J. Aust. Math. Soc. 82(1) (2007), 59-83.

[13] J. P. Pier, Amenable Locally Compact Groups, Pure and Applied Mathematics (Wiley, New York, 1984).

[14] H. Reiter and J. D. Stegeman, Classical Harmonic Analysis and Locally Compact Groups, 2nd edn, London Mathematical Society Monographs. New Series, 22 (Oxford University Press, New York, 2000).

[15] C. E. Rickart, General Theory of Banach Algebras, The University Series in Higher Mathematics (D. van Nostrand, Princeton, NJ-Toronto-London-New York, 1960).

[16] M. Skantharajah, 'Amenable hypergroups', Illinois J. Math. 36(1) (1992), 15-46.

[17] R. C. Vrem, 'Harmonic analysis on compact hypergroups', Pacific. J. Math. 85 (1979), 239-251.

MAHMOOD ALAGHMANDAN, Department of Mathematics and Statistics, University of Saskatchewan, Saskatoon, Canada SK S7N 5E6

e-mail: mahmood.a@usask.ca 